\title{
A Simple and Highly Efficient Enantioselective Synthesis of (S)-Rivastigmine
}

\author{
Veera R. Arava ${ }^{1 *}$, Laxminarasimhulu Gorentla ${ }^{1}$, Pramod K. Dubey ${ }^{2}$ \\ ${ }^{1} R \& D$ Laboratory, Suven Life Sciences Ltd., Hyderabad, India \\ ${ }^{2}$ Department of Chemistry, J. N. T. University, Hyderabad, India \\ E-mail: reddyvenis@rediffmail.com \\ Received April 1, 2011; revised May 17, 2011; accepted May 28, 2011
}

\begin{abstract}
A highly efficient and convenient procedure for the enantioselective synthesis of $(S)$-Rivastigmine, a cholinergic agent for the treatment of mild to moderate dementia of the Alzheimer's type and dementia due to Parkinson's disease, is accomplished by the treatment of versatile, readily accessible $(S)-(-)-2$-methyl2-propanesulfinamide with 3-hydroxyacetophenone. This protocol provides high yield and excellent enantiomeric excess in short step synthesis.
\end{abstract}

Keywords: Cholinergic Agent, Enantioselective, Highly Efficient, (S)-(-)-2-Methyl-2-Propane Sulfinamide, $(S)$-Rivastigmine

\section{Introduction}

Alzheimer's disease (AD) is the most common form of dementia, a severe human health threat with more than 30 million sufferers worldwide [1]. Rivastigmine, $(S)$-3[1-(dimethylamino)ethyl] phenyl ethyl (methyl) carbamate $\mathbf{1}$, is the first USFDA approved drug in the form of capsules and patches for the treatment of mild to moderate dementia of the Alzheimer's type [2-5] and for mild to moderate dementia related to Parkinson's disease [6]. In the year 2006, it has been used in more than 6 million patients worldwide. Rivastigmine works by inhibiting both butyrylcholinesterase (BuChE) and acetylcholinesterase (AChE) with the same potency, unlike donepezil 2 (which selectively inhibits acetylcholinesterase), mimantine $\mathbf{3}$ and galanthamine $\mathbf{4}$. Its pharmacological effect is selectively on the central nervous system, preferentially on the monomeric G1 isoform of $\mathrm{AChE}$ (selectivity for different isoforms of $\mathrm{BuChE}$ have not yet been investigated). The metabolism of rivastigmine is by the enzyme cholinesterase and do not depend on the P450 system in the liver, which reduces the risk of interactions with other medications [7]. The other stigmine products are pyridostigmine 5, physostigmine 6 and neostigmine 7 (Figure 1).

Rivastigmine has one chiral center with amine functionality. Therefore, attention needs to be paid to the synthesis of the correct enantiomer. (S)-enantiomer ex- hibits the desired cholinesterase inhibition, which requires the drug in enantiomerically pure form.

The insertion of chiral amine functionality through $\mathrm{N}$-sulfinylimines is a major breakthrough endeavor due to the exceptional behavior of the chiral sulfinyl group in $\mathrm{N}$-sulfinylimines, as an activator, chiral controller and useful protective group and finally recyclability [8] makes the sulfinamides extremely versatile chiral reagents (Figure 2) [9].

\section{Results and Discussion}

Our research group has been interested in utilization of these sulfinamides in industrial perspective for the development of facile chemical processes for active pharmaceutical ingredients (API's) [10]. Although several methods for the synthesis of rivastigmine and phenylcarbamate derivatives have been reported (Scheme 1), these methods suffer from limitations. Initial approaches have been developed via resolution of recemate using chiral acids [11-14] and transition metal catalysis [15,16]. Recent approaches are based on lipase catalyzed kinetic resolution [17-19], chemoenzymatic asymmetric synthesis [20] and asymmetric transfer hydrogenation [21].

In order to circumvent these difficulties and provide access to feasible industrial process, we have devised a highly efficient enantioselective synthetic route based on the report that enantiopure tert-butane-sulfinamides take 
<smiles>CCN(C)C(=O)Oc1cccc(C(C)N(C)C)c1</smiles>

(S)-Rivastigmine. 1<smiles>CN(C)C(=O)Oc1ccc[n+](C)c1</smiles>

Pyridostigmine. 5<smiles>CNC(=O)Oc1ccc2c(c1)C1(C)CCN(C)C1N2C</smiles>

Physostigmine. 6<smiles></smiles>

Neostigmine. 7<smiles>COc1cc2c(cc1OC)C(=O)C(CC1CCN(Cc3ccccc3)CC1)C2</smiles>

Donepezil. 2

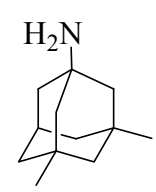

Mimantine. 3<smiles>COc1ccc2c3c1OC1CC(=CC(O)C1)C23</smiles>

Galanthamine. 4

Figure 1. Acetylcholinesterase inhibitors.<smiles>CC(C)(C)S(N)=O</smiles><smiles>CC(C)(C)S(N)=O</smiles>

(R)-tert-butanesulfinamide

(S)-tert-butanesulfinamide. 8

Figure 2. Chiral tert-butanesulfinamides.<smiles>CC(=O)c1cccc(O)c1</smiles>

9

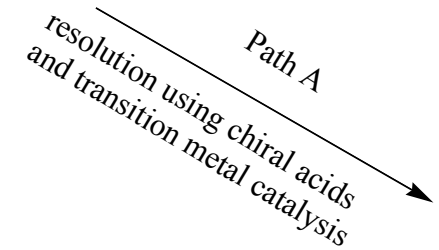<smiles>CCN(C)C(=O)Oc1cccc(C(C)=O)c1</smiles>

10<smiles>CCN(C)C(=O)Oc1cccc(C(C)N(C)C)c1</smiles>

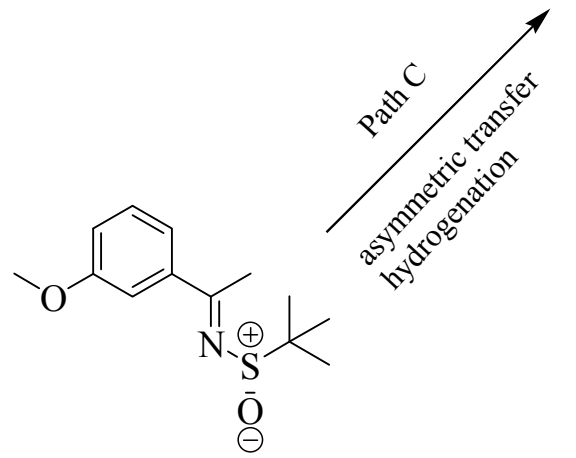

11
(S)-Rivastigmine. 1

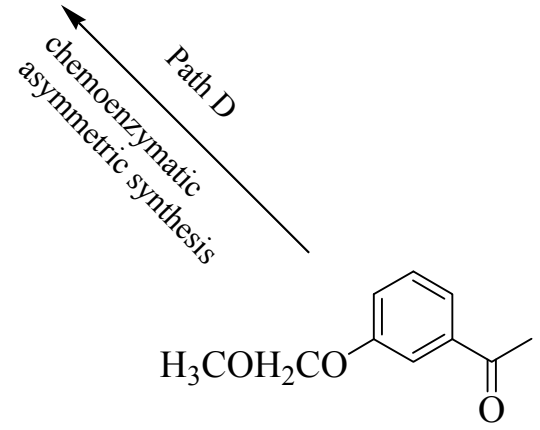

12

\section{Scheme 1}

part in the direct and enantioselective addition to aldehydes and ketones [9].
Initially we have synthesized 1 starting from 3-methoxyacetophenone 13 [22] via four step synthesis. The 
procedure includes, insitu preparation of $N$-sulfinylimine 11 from $\mathbf{1 3}$ and $(S)$-8 using $\mathrm{Ti}(\mathrm{OEt})_{4}$ as Lewis acid and water scavenger in tetrahydrofuran. Imine on reduction with sodium borohydride at $-48^{\circ} \mathrm{C}$ furnished 14. Hydrolysis of the sulfinyl group in $\mathbf{1 4}$ with dry methanolic hydrogen chloride gives the $\mathrm{HCl}$ salt $\mathbf{1 5}$ in pure form which on alkali treatment yields the chiral amine intermediate 16 in $65 \%$ yield with $>99 \%$ ee. 16 was subjected to $N, N$-dimethylation using formic acid and formaldehyde results $\mathbf{1 7}$ in $90 \%$ isolated yield. Demethylation of 17 in $48 \%$ aq. $\mathrm{HBr}$ proceeded in $87 \%$ yield of 18. Finally treatment of $\mathbf{1 8}$ with $N$-ethyl- $N$ methyl carbamoyl chloride in the presence of sodium methoxide furnished the free base of $\mathbf{1}$ in $93 \%$ isolated yield with an enantiomeric excess of $>99 \%$ (Scheme 2).

Still not satisfied with this yield and chiral purity, we set out to investigate the alternate possibilities for opti- mization of the reaction conditions and found great improvement in yield and also achieved the excellent enantiomeric excess of the desired product ( $100 \%$ ee). This time we chose $\mathbf{9}$ as starting material and synthesized $\mathbf{1}$ in two different routes. One is by condensing 1.25 equiv of $\mathrm{N}$-ethyl- $\mathrm{N}$-methylcarbamoyl chloride with 9 in the presence of $\mathrm{K}_{2} \mathrm{CO}_{3}$ (2.0 equiv) as base and ethyl acetate as solvent medium resulted $\mathbf{1 0}$ in $98.5 \%$ yield. $\mathbf{1 0}$ on condensation with 8 ( $S$-isomer) furnished the imine intermediate $19 \mathrm{insitu}$, which was reduced with sodium borohydride at $-48^{\circ} \mathrm{C}$ to get 20 . Hydrolysis of the sulfinyl group in $\mathbf{2 0}$ and alkali treatment at room temperature afforded the desired chiral amine 21 in $81 \%$ yield (Scheme 3). $N, N$-dimethylation of the amine with $98 \%$ formic acid and $35 \%$ formaldehyde solution liberates free base of 1 in $95 \%$ yield with $100 \%$ ee (Scheme 4). And the other route proceeded by the treatment of 9 with

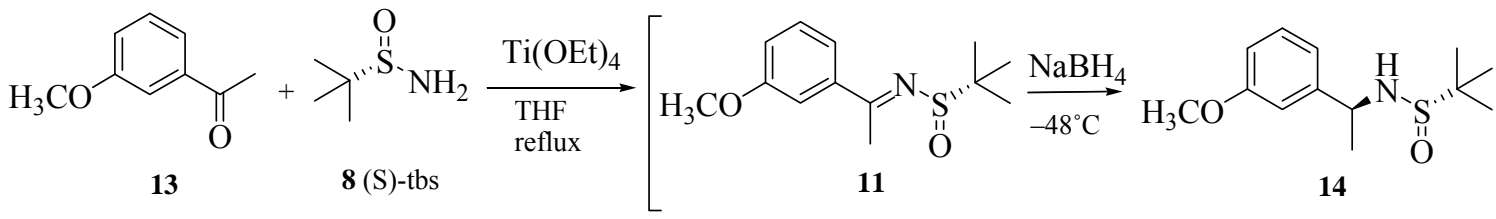<smiles>COc1cccc(C(C)N(C)CCC(C(=O)O)C(C)C(C(=O)O)C(C)N)c1</smiles><smiles>CCN(CC)C(=O)N(CC)C(=O)Cl</smiles>

Scheme 2

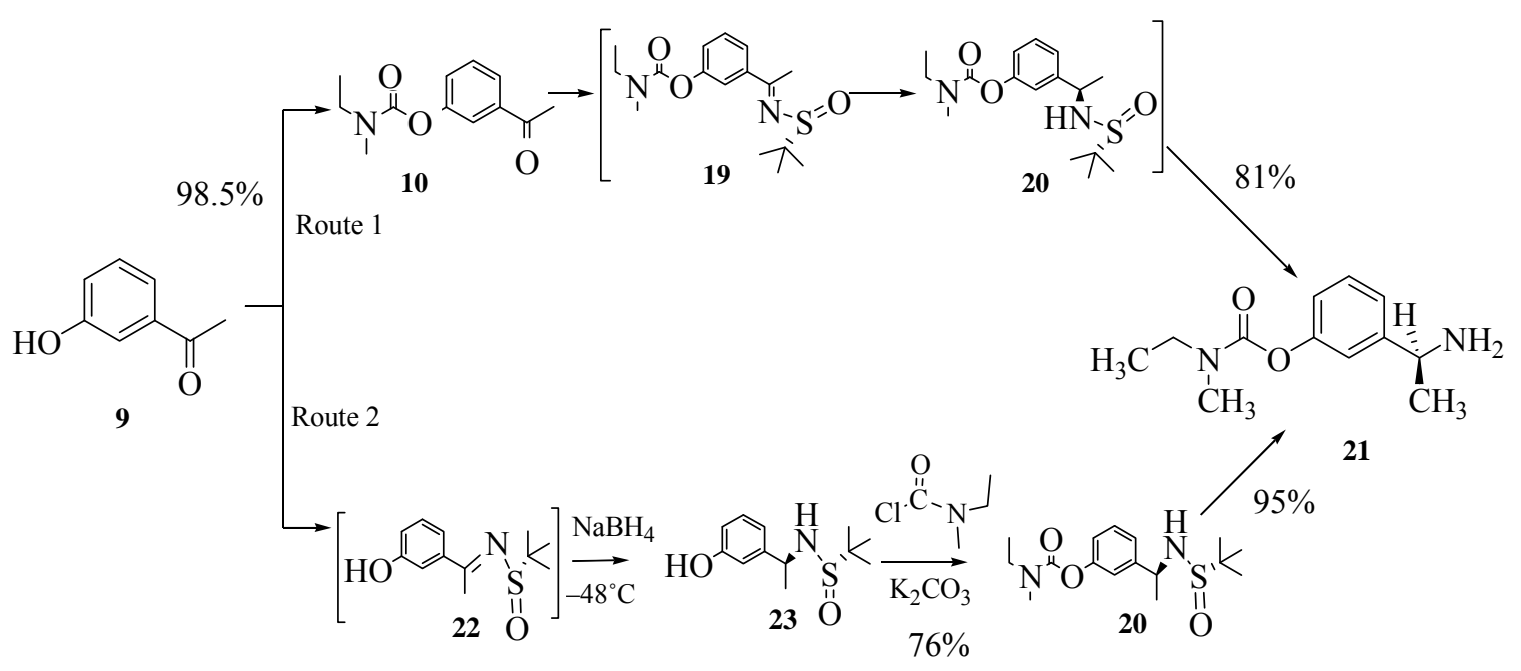

Scheme 3. Current routes of asymmetric synthesis of 21 from 9. 
<smiles>CCN(C)C(=O)Oc1cccc([C@H](C)N)c1</smiles>

21

Scheme 4

8 ( $S$-isomer) first, then reduction of the $N$-sulfinylimine 22 followed by amido-ester formation with $\mathrm{N}$-ethyl- $\mathrm{N}$ methyl carbamoyl chloride to afford 20 in $76 \%$ yield. Hydrolysis of sulfinyl group with dry methanolic hydrogen chloride and alkali treatment gives 21 base with chiral purity $>94 \%$ in $95 \%$ yield (Scheme 3 ). Finally $\mathrm{N}, \mathrm{N}$-dimethylation of amine $\mathbf{2 1}$ with formic acid and $35 \%$ formaldehyde solution furnished 1 (free base) with $98 \%$ chiral purity in $95 \%$ yield (Scheme 4 ). The chiral purity is then improved to $100 \%$ by tartarate salt preparation with $L-(+)$-tartaric acid.

\section{Experimental Section}

Experiments are conducted under nitrogen atmosphere unless stated otherwise. All solvents and reagents are reagent grade pure and used without further purification. All melting points are determined on Polmon MP_96 melting point apparatus. ${ }^{1} \mathrm{H} \&{ }^{13} \mathrm{C}$ NMR spectra are recorded using a Bruker $400 \mathrm{MHz}$ spectrometer (400 \& $100 \mathrm{MHz}$ respectively) with TMS as internal standard. Mass spectra are recorded on a Perkin-Elmer mass spectrometer operating at an ionization potential of $70 \mathrm{eV}$. IR spectra are recorded on Perkin Elmer spectrophotometer as $\mathrm{KBr}$ pellets or neat. Analytical TLC is conducted on E-Merck 60F254 aluminum-packed silicagel plates $(0.2$ $\mathrm{mm}$ ). Developed plates are visualized under UV light or Iodine chamber. Chiral HPLC spectra are recorded on Waters alliance 2695 with 2487 U. V. detector.

Typical procedure for the preparation of $(S)$-Rivastigmine (1)

\section{Route A.}

Synthesis of 3-acetylphenyl ethyl (methyl) carbamate (10): a mixture of $9(50.0 \mathrm{~g}, 0.367 \mathrm{~mol}), \mathrm{K}_{2} \mathrm{CO}_{3}$ (101.5 g, $0.734 \mathrm{~mol}$ ) and $N$-ethyl- $N$-methyl carbamoyl chloride $(56.0 \mathrm{~g}, 0.46 \mathrm{~mol})$ in ethyl acetate $(500 \mathrm{ml})$ is refluxed for 4 hours. (Completion of reaction is monitored by TLC). The reaction mixture is cooled to room temperature and washed with water $(3 \times 250 \mathrm{~mL})$, then dried over anhydrous $\mathrm{Na}_{2} \mathrm{SO}_{4}$, filtered and evaporated to get pale yellow oil of $\mathbf{1 0}$. Wt $80.0 \mathrm{~g}, 98.5 \%$ yield; HPLC Purity: 97\%; IR (Neat, $\left.v_{\max } \mathrm{cm}^{-1}\right): 1724(\mathrm{C}=\mathrm{O}), 1686$ $(\mathrm{C}=\mathrm{O}), 2974$ (aliphatic $\mathrm{CH}) ;{ }^{1} \mathrm{H}-\mathrm{NMR} \quad(400 \mathrm{MHz}$, $\left.\mathrm{CDCl}_{3}\right): \delta_{\mathrm{H}} 7.74(d, 1 \mathrm{H}, J=7.6 \mathrm{~Hz}, \mathrm{ArH}), 7.65(s, 1 \mathrm{H}$, $\operatorname{ArH}), 7.40(t, 1 \mathrm{H}, J=7.9 \mathrm{~Hz}, \mathrm{ArH}), 7.30(d, 1 \mathrm{H}, J=7.5$
$\mathrm{Hz}, \mathrm{ArH}), 3.42\left(q, 1 \mathrm{H}, J=7.0 \mathrm{~Hz}\right.$, rotamer1 $\left.\mathrm{CH}_{2}\right), 3.37$ $\left(q, 1 \mathrm{H}, J=7.0 \mathrm{~Hz}\right.$, rotamer2 $\left.\mathrm{CH}_{2}\right), 3.04(s, 1.5 \mathrm{H}$, rotamer1 $\left.\mathrm{CH}_{3}\right), 2.95\left(s, 1.5 \mathrm{H}\right.$, rotamer2 $\left.\mathrm{CH}_{3}\right), 2.55(s, 3 \mathrm{H}$, $\left.\mathrm{CH}_{3}\right), 1.21\left(t, 1.5 \mathrm{H}, J=7.0 \mathrm{~Hz}\right.$, rotamer1 $\left.\mathrm{CH}_{3}\right), 1.15(t$, $1.5 \mathrm{H}, J=7.0 \mathrm{~Hz}$, rotamer2 $\left.\mathrm{CH}_{3}\right) ;{ }^{13} \mathrm{C} \mathrm{NMR}(100 \mathrm{MHz}$, $\left.\mathrm{CDCl}_{3}\right): \delta_{\mathrm{C}} 197.08(1 \mathrm{C}, \mathrm{C}=\mathrm{O}), 153.99(d, J=15.0 \mathrm{~Hz}$, $1 \mathrm{C}, \mathrm{C}=\mathrm{O}), 151.64\left(\mathrm{C}_{\text {aryl }}\right), 138.19\left(\mathrm{C}_{\text {aryl }}\right), 129.26\left(\mathrm{C}_{\text {aryl }}\right)$, $126.57\left(\mathrm{C}_{\text {aryl }}\right), 124.93\left(\mathrm{C}_{\text {aryl }}\right), 121.47\left(\mathrm{C}_{\text {aryl }}\right), 43.98(1 \mathrm{C}$, $\mathrm{CH}_{2}$ of carbamoyl), 34.14 (rotamer1 $\mathrm{Me}_{\text {carbamoyl), }} 33.70$ (rotamer2 $\mathrm{Me}_{\text {carbamoyl }}$ ), $26.56\left(1 \mathrm{C}, \mathrm{CH}_{3}\right), 13.10$ (rotamer1 $\mathrm{Me}_{\text {carbamoyl }}$ ), 12.29 (rotamer2 $\mathrm{Me}_{\text {carbamoyl }}$ ); ESI-MS $\mathrm{m} / \mathrm{z}$ (\%): $222.0\left(\mathrm{M}^{+}, 100\right), 223.1\left(\mathrm{M}^{+}+\mathrm{H}, 20\right)$.

Synthesis of $(S)$-Ethyl-methyl-carbamic acid 3-(1-aminoethyl) phenyl ester (21): compound 10 (80.0 $\mathrm{g}, 0.36 \mathrm{~mol})$ is refluxed with $(S)$-tert-butanesulfinamide 8 (48.2 g, $0.39 \mathrm{~mol})$, titanium tetra ethoxide $(164.26 \mathrm{~g}, 0.72 \mathrm{~mol})$ in tetrahydrofuran $(800.0 \mathrm{ml})$ for 30 hours. Cool to ambient temperature and further to -48 to $-52^{\circ} \mathrm{C}$. Add $\mathrm{NaBH}_{4}(20.5 \mathrm{~g}, 0.54 \mathrm{~mol})$ in 5 portions. Stir for 3 hours to complete the reduction of imine intermediate 19. Gradually warm to $-5^{\circ} \mathrm{C}$, add methanol $(80 \mathrm{ml})$ drop wise at $-5^{\circ} \mathrm{C}$ to $0^{\circ} \mathrm{C}$ and stir for 30 minutes. Add ethyl acetate $(400 \mathrm{ml})$ and water $(500 \mathrm{ml})$. Stir for 30 minutes to warm to rt and filter off the salts. Separate the organic layer and aqueous layer is washed with ethyl acetate $(200 \mathrm{ml})$. Combined organic layers are washed successively with water $(2 \times 250 \mathrm{ml})$ and brine solution $(200 \mathrm{ml})$. The organic layer is dried over anhydrous $\mathrm{Na}_{2} \mathrm{SO}_{4}$ and evaporated under reduced pressure to get yellow oil of crude 20, which is hydrolyzed with $13 \%$ methanolic $\mathrm{HCl}(126 \mathrm{ml})$ and then alkali treatment with aq. $\mathrm{NaOH}$ at rt, extraction into ethyl acetate and concentration of the solvent afford pale yellow oil. Wt $65.0 \mathrm{~g}$, yield $81 \%$; $[\alpha]_{D}{ }^{20}-16.5^{\circ}\left(c 1.0, \mathrm{CHCl}_{3}\right)$; Chiral HPLC Purity: 99.9\%, Column: chiral cel OD-H $250 \times 4.6$ (SRC-583), mobile phase A: $n$-hexane:IPA:DEA (90:10: 0.1 , Iso: $100 \%$, concentration: $10 \mathrm{mg} / 10 \mathrm{~mL}$, diluent: ethanol, run time: $20 \mathrm{~min}$, temperature: $25^{\circ} \mathrm{C}$, flow rate: $1 \mathrm{~mL} / \mathrm{min}, \mathrm{UV}: 225 \mathrm{~nm}$, retention time: $(S)$-isomer: 12.8 min and $(R)$-isomer: 11.66 min; HPLC Purity: 98.8\%; IR (Neat, $\left.v_{\max }, \mathrm{cm}^{-1}\right): 1716(\mathrm{C}=\mathrm{O}), 2974$ (aliphatic $\mathrm{CH}$ ), $3365\left(\mathrm{NH}_{2}\right) ;{ }^{1} \mathrm{H}-\mathrm{NMR}\left(400 \mathrm{MHz}, \mathrm{CDCl}_{3}\right): \delta_{\mathrm{H}} 7.31(t, 1 \mathrm{H}$, $J=7.8 \mathrm{~Hz}, \mathrm{ArH}), 7.27(s, 1 \mathrm{H}, \mathrm{ArH}), 7.16(d, 1 \mathrm{H}, J=7.7$ $\mathrm{Hz}, \operatorname{ArH}), 6.99(d, 1 \mathrm{H}, J=7.7 \mathrm{~Hz}, \operatorname{ArH}), 4.12(q, 1 \mathrm{H}, J=$ $3.4 \mathrm{~Hz}, \mathrm{CH}), 3.46\left(q, 1 \mathrm{H}, J=7.2 \mathrm{~Hz}\right.$, rotamer1 $\left.\mathrm{CH}_{2}\right)$, 
$3.41\left(q, 1 \mathrm{H}, J=7.1 \mathrm{~Hz}\right.$, rotamer2 $\left.\mathrm{CH}_{2}\right), 3.07(s, 1.5 \mathrm{H}$, rotamer1 $\left.\mathrm{CH}_{3}\right), 2.99\left(s, 1.5 \mathrm{H}\right.$, rotamer2 $\left.\mathrm{CH}_{3}\right), 1.61(s, 2 \mathrm{H}$, $\left.\mathrm{NH}_{2}\right), 1.38\left(d, 3 \mathrm{H}, J=6.6 \mathrm{~Hz}, \mathrm{CH}_{3}\right), 1.24(t, 1.5 \mathrm{H}, J=$ $6.1 \mathrm{~Hz}$, rotamer1 $\left.\mathrm{CH}_{3}\right), 1.19(t, 1.5 \mathrm{H}, J=6.0 \mathrm{~Hz}$, rotamer2 $\left.\mathrm{CH}_{3}\right) ;{ }^{13} \mathrm{C}-\mathrm{NMR}\left(100 \mathrm{MHz}, \mathrm{CDCl}_{3}\right): \delta_{\mathrm{C}} 154.45(d$, $J=18.0 \mathrm{~Hz}, 1 \mathrm{C}, \mathrm{C}=\mathrm{O}), 151.57\left(\mathrm{C}_{\text {ary }}\right), 149.22\left(\mathrm{C}_{\text {aryl }}\right)$, $129.10\left(\mathrm{C}_{\text {aryl }}\right), 122.39\left(\mathrm{C}_{\text {aryl }}\right), 120.02\left(\mathrm{C}_{\text {aryl }}\right), 118.95\left(\mathrm{C}_{\text {ary }}\right)$, $50.95(1 \mathrm{C}, \mathrm{CH}), 43.94$ (1C, $\mathrm{CH}_{2}$ of carbamoyl), 34.11 (rotamer1 $\mathrm{Me}_{\text {carbamoyl }}$ ), 33.68 (rotamer2 $\mathrm{Me}_{\text {carbamoyl) }}$ ), 25.40 (1C, $\mathrm{CH}_{3}$ ), 13.10 (rotamer1 $\mathrm{Me}_{\text {carbamoyl) }}$ ), 12.35 (rotamer2 $\left.\mathrm{Me}_{\text {carbamoyl }}\right)$; ESI-MS $\mathrm{m} / \mathrm{z}$ (\%): $206.0\left(\mathrm{M}^{-N_{2}}, 100\right)$, 207.1 $\left(\mathrm{M}^{+}-\mathrm{NH}_{2}, 20\right), 223.1\left(\mathrm{M}^{+}+\mathrm{H}, 15\right)$.

The intermediate stages have also isolated for confirmation by analytical and spectral data.

2-Methyl propane-2-sulfinic acid [1-(3-methoxyphenyl)ethylidene]amide (19): yellow oil, yield $87 \%$; $[\alpha]_{D}{ }^{20}-0.5^{\circ}$ (c 1.0, $\mathrm{CHCl}_{3}$ ); HPLC Purity: 96.8\%; IR (Neat, $\left.v_{\max }, \mathrm{cm}^{-1}\right): 1089(\mathrm{~S}-\mathrm{O}), 1723(\mathrm{C}=\mathrm{O}), 1575(\mathrm{C}=\mathrm{N})$, 2975 (aliphatic $\mathrm{CH}) ;{ }^{1} \mathrm{H}$ NMR $\left(400 \mathrm{MHz}, \mathrm{CDCl}_{3}\right): \delta_{\mathrm{H}}$ $7.71(d, 1 \mathrm{H}, J=7.5 \mathrm{~Hz}, \mathrm{ArH}), 7.59(s, 1 \mathrm{H}, \mathrm{ArH}), 7.40(t$, $1 \mathrm{H}, J=7.9 \mathrm{~Hz}, \mathrm{ArH}), 7.25(d, 1 \mathrm{H}, J=8.0 \mathrm{~Hz}, \mathrm{ArH})$, $3.48\left(\mathrm{q}, 1 \mathrm{H}, J=7.0 \mathrm{~Hz}\right.$, rotamer1 $\left.\mathrm{CH}_{2}\right), 3.41(q, 1 \mathrm{H}, J=$ $7.0 \mathrm{~Hz}$, rotamer2 $\left.\mathrm{CH}_{2}\right), 3.08\left(s, 1.5 \mathrm{H}\right.$, rotamer1 $\left.\mathrm{CH}_{3}\right)$, $2.99\left(s, 1.5 \mathrm{H}\right.$, rotamer2 $\left.\mathrm{CH}_{3}\right), 2.74\left(s, 3 \mathrm{H}, \mathrm{CH}_{3}\right), 1.30(s$, $\left.9 \mathrm{H}, \mathrm{C}\left(\mathrm{CH}_{3}\right)_{3}\right), 1.24\left(t, 1.5 \mathrm{H}, J=7.0 \mathrm{~Hz}\right.$, rotamer1 $\left.\mathrm{CH}_{3}\right)$, $1.19\left(t, 1.5 \mathrm{H}, J=7.0 \mathrm{~Hz}\right.$, rotamer2 $\left.\mathrm{CH}_{3}\right) ;{ }^{13} \mathrm{C} \mathrm{NMR}$ $\left(100 \mathrm{MHz}, \mathrm{CDCl}_{3}\right): \delta_{\mathrm{C}} 175.27(1 \mathrm{C}, \mathrm{C}=\mathrm{N}), 154.12(d, J=$ $18.0 \mathrm{~Hz}, 1 \mathrm{C}, \mathrm{C}=\mathrm{O}), 151.52\left(\mathrm{C}_{\text {aryl }}\right), 139.93\left(\mathrm{C}_{\text {ary }}\right), 129.11$ $\left(\mathrm{C}_{\text {ary }}\right), 125.12\left(\mathrm{C}_{\text {aryl }}\right), 123.96\left(\mathrm{C}_{\text {aryl }}\right), 120.53\left(\mathrm{C}_{\text {ary }}\right), 57.55$ (1C, $\left.\mathrm{C}_{\text {t-butyl }}\right), 44.02$ (1C, $\mathrm{CH}_{2}$ of carbamoyl), 34.19 (rotamer1 $\mathrm{Me}_{\text {carbamoyl }}$ ), 33.76 (rotamer2 $\left.\mathrm{Me}_{\text {carbamoyl }}\right), 22.46$ (3C, $\left.\mathrm{C}_{\text {t-butyl }}\right), 19.77$ (1C, Me), 13.14 (rotamer1 Me $\mathrm{Me}_{\text {carbamoyl }}$ ), 12.34 (rotamer2 $\mathrm{Me}_{\text {carbamoyl) }}$ ) ESI-MS m/z (\%): $346.9\left(\mathrm{M}^{+}\right.$ + Na, 100), $325.0(\mathrm{M}+\mathrm{H}, 45), 306.9$ (20), 291 (65), 250.9 (47), 243.0 (45).

2-Methyl propane-2-sulfinic acid [1-(3-methoxyphenyl) ethyl]amide (20): yellow oil, yield $98 \%$; $[\alpha]_{D}{ }^{20}$ $+26.0^{\circ}$ (c 1.0, $\mathrm{CHCl}_{3}$ ); Chiral HPLC Purity: 99.95\%, Column: chiral cel AD-H $250 \times 4.6 \mathrm{~mm} / 5 \mu \mathrm{m}$ (SRC581), mobile phase A: $n$-hexane:IPA:TFA (90:10:0.1), Iso: $100 \%$, concentration: $10 \mathrm{mg} / 10 \mathrm{~mL}$, diluent: ethanol, run time: $35 \mathrm{~min}$, temperature: $25^{\circ} \mathrm{C}$, flow rate: 0.8 $\mathrm{mL} / \mathrm{min}, \mathrm{UV}: 215 \mathrm{~nm}$, retention time: $(S)$-isomer: 12.78 min and $(R)$-isomer: $7.72 \mathrm{~min}$; HPLC Purity: 92\%; IR (Neat, $\left.v_{\max }, \mathrm{cm}^{-1}\right)$ : $1055(\mathrm{~S}-\mathrm{O}), 1718(\mathrm{C}=\mathrm{O}), 2978$ (aliphatic $\mathrm{CH}), 3234(\mathrm{NH}) ;{ }^{1} \mathrm{H}$ NMR $\left(400 \mathrm{MHz}, \mathrm{CDCl}_{3}\right): \delta_{\mathrm{H}}$ $7.32(t, 1 \mathrm{H}, J=7.8 \mathrm{~Hz}, \mathrm{ArH}), 7.18(d, 1 \mathrm{H}, J=7.6 \mathrm{~Hz}$, ArH), $7.08(s, 1 \mathrm{H}, \mathrm{ArH}), 7.04(d, 1 \mathrm{H}, J=7.0 \mathrm{~Hz}, \mathrm{ArH})$, $4.54(q, 1 \mathrm{H}, J=3.7 \mathrm{~Hz}, \mathrm{CH}), 3.51(s, 1 \mathrm{H}, \mathrm{NH}), 3.47(q$, $1 \mathrm{H}, J=7.0 \mathrm{~Hz}$, rotamer1 $\left.\mathrm{CH}_{2}\right), 3.41(q, 1 \mathrm{H}, J=7.1 \mathrm{~Hz}$, rotamer2 $\left.\mathrm{CH}_{2}\right), 3.06\left(s, 1.5 \mathrm{H}\right.$, rotamer1 $\left.\mathrm{CH}_{3}\right), 2.98(s$, $1.5 \mathrm{H}$, rotamer2 $\left.\mathrm{CH}_{3}\right), 1.50\left(d, 3 \mathrm{H}, J=6.5 \mathrm{~Hz}, \mathrm{CH}_{3}\right), 1.25$ $\left(t, 1.5 \mathrm{H}, J=7.0 \mathrm{~Hz}\right.$, rotamer1 $\left.\mathrm{CH}_{3}\right), 1.20(s, 9 \mathrm{H}$,
$\left.\mathrm{C}\left(\mathrm{CH}_{3}\right)_{3}\right), 1.19\left(t, 1.5 \mathrm{H}, J=7.0 \mathrm{~Hz}\right.$, rotamer2 $\left.\mathrm{CH}_{3}\right) ;{ }^{13} \mathrm{C}$ NMR $\left(100 \mathrm{MHz}, \mathrm{CDCl}_{3}\right): \delta_{\mathrm{C}} 154.20(d, J=18.0 \mathrm{~Hz}, 1 \mathrm{C}$, $\mathrm{C}=\mathrm{O}), \quad 151.59 \quad\left(\mathrm{C}_{\text {aryl }}\right), \quad 145.27 \quad\left(\mathrm{C}_{\text {aryl }}\right), 129.39 \quad\left(\mathrm{C}_{\text {aryl }}\right)$, $123.28\left(\mathrm{C}_{\text {aryl }}\right), 121.12\left(\mathrm{C}_{\text {aryl }}\right), 119.85\left(\mathrm{C}_{\text {aryl }}\right), 55.33(1 \mathrm{C}$, $\left.\mathrm{C}_{\text {t-butyl }}\right), 53.51(1 \mathrm{C}, \mathrm{CH}), 43.92\left(1 \mathrm{C}, \mathrm{CH}_{2}\right.$ of carbamoyl), 34.08 (rotamer1 $\mathrm{Me}_{\text {carbamoyl) }}$ ) 33.68 (rotamer2 $\mathrm{Me}_{\text {carbamoyl), }}$ $29.14(1 \mathrm{C}, \mathrm{Me}), 22.48$ (3C, $\mathrm{C}_{\text {t-butyl) }}, 13.09$ (rotamer1 Mecarbamoyl), 12.32 (rotamer2 $\mathrm{Me}_{\text {carbamoyl); }}$ ) ESI-MS $m / z$ (\%): $348.9\left(\mathrm{M}^{+}+\mathrm{Na}, 90\right), 327.0(\mathrm{M}+\mathrm{H}, 100), 293.0$ (23), 252.9 (54), 245.0 (26), 194.0 (80).

\section{Route B.}

Synthesis of 2-Methyl propane-2-sulfinic acid [1-(3hydroxyphenyl)ethyl]amide (23):

3-Hydroxyacetophenone $(10.0 \mathrm{~g}, 0.066 \mathrm{~mol})$ is refluxed with $(S)-8(8.07 \mathrm{~g}, 0.066 \mathrm{~mol})$, titanium tetra ethoxide $(30.4 \mathrm{~g}, 0.133 \mathrm{~mol})$ in THF $(100 \mathrm{ml})$ for $30 \mathrm{hr}$. Cool to ambient temperature and further to -48 to $-52^{\circ} \mathrm{C}$ and added $\mathrm{NaBH}_{4}(3.8 \mathrm{~g}, 0.099 \mathrm{~mol})$ in 4 portions. Stir for 3 hours to complete the reduction of imine intermediate 22. Gradually warm to $-5^{\circ} \mathrm{C}$ and added methanol $(10$ $\mathrm{ml}$ ) drop wise at $-5^{\circ} \mathrm{C}$ to $0^{\circ} \mathrm{C}$. Stir for 30 minutes, added ethyl acetate $(50 \mathrm{ml})$ and water $(100 \mathrm{ml})$. Stir for 30 minutes to warm to rt and filtered off the salts. Separate organic layer and aqueous layer is washed with ethyl acetate $(50 \mathrm{ml})$. Combined organic layers are washed successively with water $(2 \times 50 \mathrm{~mL})$ and brine solution $(30 \mathrm{ml})$. The organic layer is dried over anhydrous $\mathrm{Na}_{2} \mathrm{SO}_{4}$ and evaporated to afford pale yellow solid of 23 . Wt $13.7 \mathrm{~g}$; yield $77.6 \%$; mp $140^{\circ} \mathrm{C}-144^{\circ} \mathrm{C} ;[\alpha]_{D}{ }^{23}+$ $25.5^{\circ}$ (c 1.0, MeOH); Chiral HPLC: $94.76 \%$, Column: chiral pak AD-H $0.46 \mathrm{~cm} \times 25 \mathrm{~cm}($ SRC-581), mobile phase A: $n$-hexane:IPA:DEA (90:10:0.1), Iso: $100 \%$, concentration: $50 \mathrm{mg} / 10 \mathrm{~mL}$, diluent: ethanol, run time: $45 \mathrm{~min}$, temperature: $25^{\circ} \mathrm{C}$, flow rate: $1 \mathrm{~mL} / \mathrm{min}$, UV: $230 \mathrm{~nm}$, retention time: $(S)$-isomer: $24.87 \mathrm{~min}$ and $(R)$-isomer: $8.13 \mathrm{~min}$; IR $\left(\mathrm{KBr}, \mathrm{cm}^{-1}\right): 3277(\mathrm{NH}), 3086$ (OH), 2972 (aliphatic CH), 1017 (S-O); ${ }^{1} \mathrm{H}$ NMR (400MHz, CDCl3): $\delta_{\mathrm{H}} 8.2($ br s, $1 \mathrm{H}, \mathrm{OH}), 7.15(t, 1 \mathrm{H}, J$ $=8.0 \mathrm{~Hz}, \mathrm{ArH}), 6.81(d, 1 \mathrm{H}, J=7.7 \mathrm{~Hz}, \mathrm{ArH}), 6.77(s$, $1 \mathrm{H}, \mathrm{ArH}), 6.76(d, 1 \mathrm{H}, J=7.5 \mathrm{~Hz}, \mathrm{ArH}), \delta 4.41(q, 1 \mathrm{H}, J$ $=3.5 \mathrm{~Hz}, \mathrm{CH}), \delta 3.64(s, 1 \mathrm{H}, \mathrm{NH}), 1.46(d, 3 \mathrm{H}, J=6.5$ $\left.\mathrm{Hz}, \mathrm{CH}_{3}\right), 1.26\left(s, 9 \mathrm{H}, \mathrm{C}\left(\mathrm{CH}_{3}\right)_{3}\right) ;{ }^{13} \mathrm{C}$ NMR $(100 \mathrm{MHz}$, DMSO-d $\left._{6}\right): \delta_{\mathrm{C}} 157.57\left(1 \mathrm{C}, \mathrm{C}_{\text {aryl }}\right), 147.13\left(1 \mathrm{C}, \mathrm{C}_{\text {aryl }}\right)$, $129.41\left(1 \mathrm{C}, \mathrm{C}_{\text {aryl }}\right), 117.50\left(1 \mathrm{C}, \mathrm{C}_{\text {aryl }}\right), 114.07\left(1 \mathrm{C}, \mathrm{C}_{\text {aryl }}\right)$, $113.77\left(1 \mathrm{C}, \mathrm{C}_{\text {aryl }}\right), 55.44\left(1 \mathrm{C}, \mathrm{C}_{\text {t-butyl }}\right), 54.47(1 \mathrm{C}, \mathrm{CH})$, $24.50\left(1 \mathrm{C}, \mathrm{CH}_{3}\right), 22.97\left(3 \mathrm{C}, \mathrm{C}_{\text {t-butyl }}\right)$; ESI-MS $\mathrm{m} / \mathrm{z}(\%)$ : $242.4(\mathrm{M}+\mathrm{H}, 100), 168.3$ (50), 125.1 (10).

The intermediate stages have also isolated for confirmation by analytical and spectral data.

2-Methyl propane-2-sulfinic acid [1-(3-hydroxyphenyl)ethylidene]amide (22): yellow crystals, yield $77.6 \%$; mp $137^{\circ} \mathrm{C}-142^{\circ} \mathrm{C} ;[\alpha]^{23}{ }_{D}+3.0^{\circ}(c 1.0, \mathrm{MeOH})$; Chiral HPLC purity: $99.98 \%$, Column: chiral pak AD-H 
$0.46 \mathrm{~cm} \times 25 \mathrm{~cm}($ SRC-581), mobile phase A: $n$-hexane: IPA:DEA (90:10:0.1), Iso: 100\%, concentration: 5 $\mathrm{mg} / 10 \mathrm{~mL}$, diluent: ethanol, run time: $35 \mathrm{~min}$, temperature: $25^{\circ} \mathrm{C}$, flow rate: $1 \mathrm{~mL} / \mathrm{min}, \mathrm{UV}: 254 \mathrm{~nm}$, retention time: $(S)$-isomer: $16.16 \mathrm{~min}$ and $(R)$-isomer: $12.55 \mathrm{~min}$; IR $(\mathrm{KBr}$, $\left.\mathrm{cm}^{-1}\right)$ : $3180(\mathrm{OH}), 2987$ (aliphatic $\left.\mathrm{CH}\right), 1597(\mathrm{C}=\mathrm{N}), 1028$ $(\mathrm{S}-\mathrm{O}) ;{ }^{1} \mathrm{H}$ NMR $(400 \mathrm{MHz}, \mathrm{CDCl} 3)$ : $\delta_{\mathrm{H}} 7.72(\mathrm{br} \mathrm{s}, 1 \mathrm{H}$, $\mathrm{OH}), 7.43(s, 1 \mathrm{H}, \mathrm{ArH}), 7.32(d, 1 \mathrm{H}, J=7.4 \mathrm{~Hz}, \mathrm{ArH})$, $7.23(t, 1 \mathrm{H}, J=7.9 \mathrm{~Hz}, \mathrm{ArH}), 7.01(d, 1 \mathrm{H}, J=7.9 \mathrm{~Hz}$, $\mathrm{ArH}), 2.68\left(s, 3 \mathrm{H}, \mathrm{CH}_{3}\right), 1.32\left(s, 9 \mathrm{H}, \mathrm{C}\left(\mathrm{CH}_{3}\right)_{3}\right) ;{ }^{13} \mathrm{C} \mathrm{NMR}$ $\left(100 \mathrm{MHz}, \mathrm{DMSO}-\mathrm{d}_{6}\right): \delta_{\mathrm{C}} 176.82(1 \mathrm{C}, \mathrm{C}=\mathrm{N}), 157.78(1 \mathrm{C}$, $\left.\mathrm{C}_{\text {aryl }}\right), 140.01$ (1C, $\left.\mathrm{C}_{\text {aryl }}\right), 129.98$ (1C, $\left.\mathrm{C}_{\text {aryl }}\right), 119.34$ (1C, $\left.\mathrm{C}_{\text {aryl }}\right), 118.52\left(1 \mathrm{C}, \mathrm{C}_{\text {aryl }}\right), 113.92\left(1 \mathrm{C}, \mathrm{C}_{\text {aryl }}\right), 57.08(1 \mathrm{C}$, $\left.\mathrm{C}_{\text {t-butyl }}\right), 22.37$ (3C, $\left.\mathrm{C}_{\text {t-butyl }}\right), 20.03\left(1 \mathrm{C}, \mathrm{CH}_{3}\right)$; ESI-MS $\mathrm{m} / \mathrm{z}$ (\%): $240.3(\mathrm{M}+\mathrm{H}, 65), 184.2$ (100), 166.1 (82).

Synthesis of $(S)$-Ethyl methyl carbamic acid 3-(1aminoethyl)phenyl ester (21): a mixture of 23 (13.0 g, $0.053 \mathrm{~mol}), \mathrm{K}_{2} \mathrm{CO}_{3}(14.9 \mathrm{~g}, 0.107 \mathrm{~mol})$ and $N$-ethyl$\mathrm{N}$-methyl carbamoyl chloride $(8.18 \mathrm{~g}, 0.067 \mathrm{~mol})$ in ethyl acetate $(130 \mathrm{ml})$ is refluxed for 4 hours. Cool to room temperature and washed with water $(3 \times 130 \mathrm{~mL})$, dried over anhydrous $\mathrm{Na}_{2} \mathrm{SO}_{4}$, filtered and evaporated under reduced pressure to get pale yellow oil of 20 (17.37 g, $99 \%$ yield), which is hydrolyzed with $13 \%$ methanolic $\mathrm{HCl}(18 \mathrm{ml})$ and then alkali treatment with aq. $\mathrm{NaOH}$ at $\mathrm{rt}$, extraction into ethyl acetate and evaporation of the solvent under reduced pressure afford pale yellow oil of $\mathbf{2 1}$. Wt $11.25 \mathrm{~g}$, yield $95 \%$.

Synthesis of (S)-Rivastigmine (1): the pale yellow oil $21(65.0 \mathrm{~g}, 0.43 \mathrm{~mol})$ is dissolved in formic acid $(198.0 \mathrm{~g}$, $4.3 \mathrm{~mol})$, formaldehyde $(44.0 \mathrm{~g}, 3.4 \mathrm{~mol}$ (35\% solution in water) and water $(650 \mathrm{ml})$. Heated to reflux temperature $\left(95^{\circ} \mathrm{C}-100^{\circ} \mathrm{C}\right)$ and stirred for $5 \mathrm{hr}$. Cool to ambient temperature; wash with ethyl acetate $(2 \times 130 \mathrm{ml})$ to remove the impurities. Aqueous layer is separated, adjusted $\mathrm{pH}$ to 10 with $20 \%$ aq. $\mathrm{NaOH}$ solution $(758 \mathrm{ml})$ and extracted with ethyl acetate $(2 \times 325 \mathrm{ml})$. The combined organic layers are washed with brine solution (100 $\mathrm{ml}$ ), dried over anhydrous sodium sulfate and evaporated under reduced pressure at $45^{\circ} \mathrm{C}-50^{\circ} \mathrm{C}$ to get pale yellow oil. Wt $78.4 \mathrm{~g}$; yield $95 \%$; $[\alpha]_{D}{ }^{20}-35.2^{\circ}$ (c 1.0, $\left.\mathrm{CHCl}_{3}\right)$ (lit $[6]:[\alpha]_{D}{ }^{20}-33.9^{\circ}\left(c 1.0, \mathrm{CHCl}_{3}\right)$; Chiral HPLC Purity $100 \%$, Column: chiral pak OD-H $250 \times 4.6 \mathrm{~mm}$, mobile phase A: $n$-hexane:IPA:TFA (80:20:0.1), Iso: $100 \%$, concentration: $10 \mathrm{mg} / 25 \mathrm{~mL}$, diluent: ethanol, run time: 25 minutes, temperature: $25^{\circ} \mathrm{C}$, flow rate: 0.8 $\mathrm{mL} / \mathrm{min}, \mathrm{UV}: 215 \mathrm{~nm}$, retention time: $(S)$-isomer: 14.41 min and $(R)$-isomer: $9.54 \mathrm{~min}$; IR (Neat, $\left.v_{\max }, \mathrm{cm}^{-1}\right)$ : 2975 (aliphatic $\mathrm{CH}), 1725(\mathrm{C}=\mathrm{O})$; ${ }^{1} \mathrm{H}$ NMR $(400 \mathrm{MHz}$, $\left.\mathrm{CDCl}_{3}\right): \delta_{\mathrm{H}} 7.28(t, 1 \mathrm{H}, J=7.8 \mathrm{~Hz}, \mathrm{ArH}), 7.10(d, 1 \mathrm{H}, J$ $=7.8 \mathrm{~Hz}, \mathrm{ArH}), 7.05(\mathrm{~s}, 1 \mathrm{H}, \mathrm{ArH}), 7.00(d, 1 \mathrm{H}, J=7.8$ $\mathrm{Hz}, \mathrm{ArH}), 3.44\left(q, 1 \mathrm{H}, J=7.5 \mathrm{~Hz}, \mathrm{CH}_{2}\right.$ rotamer 1$), 3.41$ $\left(q, 1 \mathrm{H}, J=7.5 \mathrm{~Hz}, \mathrm{CH}_{2}\right.$ rotamer 2$), 3.23(q, 1 \mathrm{H}, J=6.6$
$\mathrm{Hz},-\mathrm{CH}), 3.05\left(s, 1.5 \mathrm{H}, \mathrm{CH}_{3}\right.$ rotamer 1$), 2.97(s, 1.5 \mathrm{H}$, $\mathrm{CH}_{3}$ rotamer 2), $2.19\left(s, 6 \mathrm{H}, \mathrm{N}\left(\mathrm{CH}_{3}\right) 2\right), 1.34(d, 3 \mathrm{H}, J=$ $\left.6.7 \mathrm{~Hz}, \mathrm{CH}_{3}\right), 1.23\left(t, 1.5 \mathrm{H}, J=7.2 \mathrm{~Hz}, \mathrm{CH}_{3}\right.$ rotamer 1), $1.17\left(t, 1.5 \mathrm{H}, J=7.2 \mathrm{~Hz}, \mathrm{CH}_{3}\right.$ rotamer 2$) ;{ }^{13} \mathrm{C} \mathrm{NMR}$ $\left(100 \mathrm{MHz}, \mathrm{CDCl}_{3}\right): \delta_{\mathrm{C}} 154.41(d, J=18.0 \mathrm{~Hz}, 1 \mathrm{C}, \mathrm{C}=$ $\mathrm{O}), 151.41\left(\mathrm{C}_{\text {aryl }}\right), 145.63\left(\mathrm{C}_{\text {aryl }}\right), 128.77\left(\mathrm{C}_{\text {aryl }}\right), 124.11$ $\left(\mathrm{C}_{\text {aryl }}\right), 120.60\left(\mathrm{C}_{\text {aryl }}\right), 120.14\left(\mathrm{C}_{\text {aryl }}\right), 65.53(1 \mathrm{C}, \mathrm{CH})$, 43.92 (1C, $\mathrm{CH}_{2}$ of carbamoyl), 43.11, 34.09 (rotamer1 $\mathrm{Me}_{\text {carbamoyl }}$ ), 33.67 (rotamer2 $\left.\mathrm{Me}_{\text {carbamoyl }}\right), 19.98$ (1C, Me), 13.12 (rotamer1 $\mathrm{Me}_{\text {carbamoyl), }} 12.37$ (rotamer2 $\mathrm{Me}_{\text {carbamoyl) }}$ ); ESI-MS $m / z(\%)$ : $251.2(\mathrm{M}+\mathrm{H}, 100), 206.2$ (60).

Preparation of $(S)-(+)$-rivastigmine hydrogentartarate (1): $(S)$-(-)-rivastigmine 1 (4.5 g) and $L-(+)$ tartaric acid $(2.7 \mathrm{~g})$ are dissolved in anhydrous ethanol $(12.5 \mathrm{~mL})$ at $60^{\circ} \mathrm{C}$. Ethyl acetate $(63 \mathrm{~mL})$ is added at the same temperature and stirred for 10 minutes. Cool the solution to room temperature and to crystallize at $+5^{\circ} \mathrm{C}$ for a period of 12 hours. The precipitated solid is collected through filtration and washed the cake with ethyl acetate $(10 \mathrm{~mL})$. Dry the solid at $50^{\circ} \mathrm{C}$ under vacuum to afford $6.7 \mathrm{~g}$ of the desired product. Yield $92 \% ;[\alpha]_{D}{ }^{20}+$ $4.6^{\circ}$ (c 5.0, ethanol); mp $117^{\circ} \mathrm{C}-120^{\circ} \mathrm{C}$; Chiral HPLC Purity: $100 \%$ ee.

\section{Conclusions}

In summary, a high yielding stereoselective and short synthesis of $(S)$-Rivastigmine is described in two ways with an overall isolated yield of $76 \%$ and $70 \%$ respectively via 3 step procedure starting from 3-hydroxyacetophenone, a commercially available intermediate. [23]

\section{Acknowledgements}

We greatly acknowledge our thanks to Mr. Venkat Jasti, CEO for his excellent support, Mr. K. R. S. Sharma for Chiral HPLC method development and the analytical group of SUVEN for providing spectral data.

\section{References}

[1] N. C. Berchtold and C. W. Cotman, "Evolution in the Conceptualization of Dementia and Alzheimer's Disease: Greco-Roman Period to the 1960s," Neurobiology of Aging. Vol. 19, No. 3, 1998, pp. 173-189. doi:10.1016/S0197-4580(98)00052-9

[2] J. Corey-Bloom, R. Anand and J. Veach, "A Randomized trial Evaluating the Efficacy and Safety of ENA 713 (Rivastigmine Tartarate), a New Acetylcholinesterase Inhibitor, in Patients with Mild to Moderately Severe Alzheimer's Disease," International Journal of Geriatric Psychopharmacology, Vol. 1, No. 2, 1998, pp. 55-65.

[3] M. Rosler, R. Anand, A. Cicin-Sain, S. Gauthier, Y. Agid, P. Dal-Bianco, H. B. Stähelin, R. Hartman and M. 
Gharabawi, "Efficacy and Safety of Rivastigmine in Patients with Alzheimer's Disease: International Randomized Controlled Trial," British Medical Journal, Vol. 318, No. 7184, 1999, pp. 633-640.

[4] S. I. Finkel, "Effects of Rivastigmine on Behavioral and Psychological Symptoms of Dementia in Alzheimer's Disease," Clinic Therapeutics, Vol. 26, No. 7, 2004, pp. 980-990. doi:10.1016/S0149-2918(04)90172-5

[5] M. Rosler, W. Retz, P. Retz-Junginger and H. J. Dennler, "Effects of Two-Year Treatment with the Cholinesterase Inhibitor Rivastigmine on Behavioural Symptoms in Alzheimer's Disease," Behavioral Neurology, Vol. 11, No. 4, 1998, pp. 211-216.

[6] M. Emre, D. Aarsland and A. Albanese, "Rivastigmine for Dementia Associated with Parkinson's Disease," The New England Journal of Medicine, Vol. 315, 2004, pp. 2509-2518. doi:10.1056/NEJMoa041470

[7] C. Gabelli, "Rivastigmine: An Update on Therapeutic Efficacy in Alzheimer's Disease and Other Conditions," Current Medical Research \& Opinion, Vol. 19, No. 2, 2003, pp. 69-82.

[8] M. Wakayama and J. A. Ellman, "Recycling the Tert-Butanesulfinyl Group in the Synthesis of Amines Using Tert-Butanesulfinamide," Journal of Organic Chemistry, Vol. 74, No. 4, 2009, pp. 2646-2650. doi:10.1021/j09001883

[9] M. T. Robak, M. A. Herbage and J. A. Ellman, "Synthesis and Applications of Tert-Butanesulfinamide," Chemical Reviews, Vol. 110, No. 6, 2010, pp. 3600-3740. doi:10.1021/cr900382t

[10] A. V. Reddy, S. U. B. Rao, G. L. Narasimha and P. K. Dubey, "Improved Process for the Preparation of Tamsulosin Hydrochloride," Synthetic Communications, Vol. 39, No. 8, 2009, pp. 1451-1456. doi:10.1080/00397910802519216

[11] A. Gaitonde, M. Mangle and S. R. Pawar, "Novel Process for the Preparation of Aminoalkyl Phenyl Carbamates," Chemical Abstracts, Vol. 143, 2005, Article ID: 77963.

[12] H. Stepankova, J. Hajicek and S. Simek, "A Method of Production of (-)-(S)-3-[1-(Dimethylamino)ethyl] phenyl$N$-ethyl- $N$-methylcarbamate," Chemical Abstracts, Vol. 142, 2004, Article ID: 6315.

[13] M. J. V. Garrido, A. M. Montserrat and M. J. Juarez, "Method of Obtaining Phenyl Carbamates," 2007, Chemical Abstracts, Vol. 146, 2007, Article ID: 206113.

[14] J. Feng, W.-M. Chen and P.-H. Sun, "Synthesis of S-(+)-Rivastigmine Hydrogen Tartarate," Journal of
Southern Medical University, Vol. 27, No. 2, 2007, pp. 177-180.

[15] A. A. Boezio, J. Pytkowicz, A. Cote and A. B. Charette, "Asymmetric, Catalytic Synthesis of $\alpha$-Chiral Amines Using a Novel Bis(Phosphine)Monoxide Chiral Ligand," Journal of the American Chemical Society, Vol. 125, No. 47, 2003, pp. 14260-14261. doi:10.1021/ja038291+

[16] M. Hu, F. L. Zhang and M. H. Xie, "Novel Convenient Synthesis of Rivastigmine," Synthetic Communications, Vol. 39, No. 9, 2009, pp. 1527-1533. doi:10.1080/00397910802531948

[17] J. M. Sanchez, M. R. Mata, E. Busto, V. G. Fernandez and V. Gotor, "Chemoenzymatic Synthesis of Rivastigmine Based on Lipase-Catalyzed Processes," The Journal of Organic Chemistry, Vol. 74, No. 15, 2009, pp. 5304-5310. doi:10.1021/jo900784g

[18] K. Han, C. Kim, J. Park and M.-J. Feng, "Chemoenzymatic Synthesis of Rivastigmine via Dynamic Kinetic Resolution as a Key Step," The Journal of Organic Chemistry, Vol. 75, No. 9, 2010, pp. 3105-3108. doi:10.1021/j09027374

[19] K. A. Kumar, M. A. Reddy, T. S. Kumar, B. V. Kumar, K. B. Chandrasekhar, P. R. Kumar and M. Pal, "Achiral Bis-Imine in Combination with $\mathrm{CoCl}_{2}$ : A Remarkable Effect on Enantioselectivity of Lipase-Mediated Acetylation of Racemic Secondary Alcohol," Beilstein Journal of Organic Chemistry, Vol. 6, 2010, pp. 1174-1179. doi:10.3762/bjoc.6.134

[20] M. Fuchs, D. Koszelewski, K. Tauber, W. Kroutil and K. Faber, "Chemoenzymatic Asymmetric Total Synthesis of (S)-Rivastigmine Using $\omega$-Transaminases," Chemical Communications, Vol. 46, No. 30, 2010, pp. 5500-5502. doi:10.1039/c0cc00585a

[21] D. Guijarro, O. Pablo and M. Yus, "Asymmetric Synthesis of Chiral Primary Amines by Transfer Hydrogenation of $\mathrm{N}$-(tert-Butanesulfinyl)ketimines," The Journal of Organic Chemistry, Vol. 75, No. 15, 2010, pp. 5265-5270. doi:10.1021/jo101057s

[22] A. V. Reddy, G. Laxminarasimhulu and P. K. Dubey, "Facile Enantioselective Synthesis of $(S)$-Rivastigmine \& (+)-NPS-R-568 a Calcimimetic Agent," Der Pharma Chemica, Vol. 3, No. 1, 2011, pp. 426-433.

[23] A. V. Reddy, "Developing Chemical Processes for Active Pharmaceutical Ingredients (API's)," 3rd International Conference and Exhibition, Mumbai, 10-11 January 2011, Abstract No. 3. 